OPEN ACCESS

Edited by:

Hector Wing Hong Tsang, Hong Kong Polytechnic University,

Hong Kong, China

Reviewed by:

Wendy Wing Yan So, Hong Kong Polytechnic University,

Hong Kong, China

Moises Betancort Montesinos,

University of La Laguna, Spain

${ }^{*}$ Correspondence: Bente Storm Mowatt Haugland bente.haugland@uib.no

Specialty section

This article was submitted to Psychology for Clinical Settings, a section of the journal

Frontiers in Psychology

Received: 06 July 2021 Accepted: 03 September 2021 Published: 27 September 2021

Citation:

Haugland BSM and Wergeland GJ (2021) Commentary: Evaluating Delivery of a CBT-Based Group Intervention for Schoolchildren With Emotional Problems: Examining the

Reliability and Applicability of a Video-Based Adherence and Competence Measure.

Front. Psychol. 12:737095. doi: 10.3389/fpsyg.2021.737095

\section{Commentary: Evaluating Delivery of a CBT-Based Group Intervention for Schoolchildren With Emotional Problems: Examining the Reliability and Applicability of a Video-Based Adherence and Competence Measure}

\author{
Bente Storm Mowatt Haugland ${ }^{1 *}$ and Gro Janne Wergeland ${ }^{2,3}$ \\ ${ }^{1}$ Department of Clinical Psychology, University of Bergen, Bergen, Norway, ${ }^{2}$ Division of Psychiatry, Department of Child and \\ Adolescent Psychiatry, Haukeland University Hospital, Bergen, Norway, ${ }^{3}$ Department of Clinical Medicine, Faculty of \\ Medicine, University of Bergen, Bergen, Norway
}

Keywords: adherence, competence, indicated prevention, youth, anxiety, group CBT

\section{A Commentary on}

Evaluating Delivery of a CBT-Based Group Intervention for Schoolchildren With Emotional Problems: Examining the Reliability and Applicability of a Video-Based Adherence and Competence Measure

by Rasmussen, L.-M. P., Patras, J., Handegård, B. H., Neumer, S.-P., Martinsen, K. D., Adolfsen, F., Sund, A. M., and Martinussen, M. (2021). Front. Psychol. 12:702565. doi: 10.3389/fpsyg.2021.702565

\section{EVALUATING ADHERENCE AND COMPETENCE IN AN INDICATED PREVENTION STUDY}

In a recent article by Rasmussen et al. (2021) the reliability and applicability of a measure on treatment adherence and competence in cognitive behavioral therapy (CBT) for youth was examined. The study was part of a Norwegian multi-site randomized controlled trial (RCT) on indicated preventive group-intervention for youth with symptoms of anxiety and depression, using the intervention EMOTION: Kids Coping with Anxiety and Depression. Therapists from community services (e.g., psychologists, school health nurses, counselors, educators) delivered the intervention. The measure applied to evaluate therapist adherence and competence was the Competence and Adherence Scale for CBT (CAS-CBT; Bjaastad et al., 2016).

CAS-CBT is an 11-item observation-based scale designed to assess adherence and competence in delivering CBT for youth with anxiety. The measure assesses CBT structure, process, and relational skills, in addition to the two main goals of each session in the intervention. Psychometric qualities of the scale have been examined previously in clinical samples (e.g., Bjaastad et al., 2016; Harstad et al., 2021). The scoring of CAS-CBT is usually based on video-recordings of therapy sessions, which was also the case in the Rasmussen et al. study.

After investigating the reliability of CAS-CBT, Rasmussen et al. (2021) found the scale to be useful, although they also identified limitations and suggested improvements in the measure. We 
find the Rasmussen et al. (2021) study to be well-conducted and have no objections regarding their conclusions. Their discussion about the need to consider treatment fidelity in youth mental health and the emphasis on examining treatment fidelity with therapists delivering indicated prevention is particularly important.

We are pleased that the CAS-CBT measure was used and that other research groups are evaluating the scale-the first author of this commentary is co-author of the CAS- CBT. However, in their article Rasmussen et al. (2021) argued that this was the first study to apply CAS-CBT in group CBT within a prevention setting, and with therapists not working in regular clinical practice delivering the intervention. They stated that besides their own study only three studies had used CAS-CBT to evaluate therapist adherence and competence-and these have all been conducted within clinical settings. Finally, they emphasized the need for research on adherence and competence in interventions within the preventive field-arguing that youth recruited to prevention studies are different from youth involved in clinical trials. They claimed that resources to support implementation in community settings are often more limited compared to clinical settings, and that assessments of adherence and competence therefore often are omitted from prevention studies.

\section{MISSING A PREVIOUS INDICATED PREVENTION STUDY APPLYING CAS-CBT}

Regretfully, Rasmussen and colleagues failed to recognize a previous large RCT where CAS-CBT was used to assess adherence and competence in indicated preventive CBT for youth anxiety delivered in group format (see Haugland et al., 2017, 2020; Husabo et al., 2021). In this RCT the CBT was delivered primarily by community providers employed by school health services or primary health services (e.g., school health nurses, community psychologists). Although the participants in the Haugland et al. study 2020 were somewhat older (mean age 14.0 years, $\mathrm{SD}=0.84)$ compared to the Rasmussen et al. (2021) study (mean age 10.1, SD 0.90), both studies were conducted within the same context and cultural setting (i.e., in schools in Norway).

There are interesting differences in methods between Rasmussen et al. (2021) and Haugland et al. (2020). These differences include student raters in Rasmussen et al. (2021) vs. experienced CBT therapists, including the CAS-CBT developers, in Haugland et al. (2020); recording of $20 \%$ of the sessions in the Rasmussen et al. study vs. recording of all sessions in the Haugland et al. study; and differences in sessions between the interventions (an extensive 20 session program in Rasmussen et al., 2021 vs. a brief 5-session and a standard 10-session program in Haugland et al., 2020). These differences could have

\section{REFERENCES}

Bjaastad, J. F., Haugland, B. S., Fjermestad, K. W., Torsheim, T., Havik, O. E., Heiervang, E. R., et al. (2016). Competence and adherence scale for cognitive behavioral therapy (CAS-CBT) for anxiety disorders in youth: psychometric properties. Psychol. Assess. 28, 908-916. doi: 10.1037/pas0000230 broadened the discussion of the findings in Rasmussen et al. (2020). For example, Haugland et al. (2020) reported higher levels of adherence and competence for the brief compared to the longer program, suggesting that it is easier for novice CBT providers to achieve fidelity in simplified and less flexible interventions (Husabo et al., 2021). This is supporting a point made by Rasmussen et al. that EMOTION is a comprehensive intervention, and that this could have had an impact on program fidelity.

Both studies found CAS-CBT to be a useful measure for settings outside clinical treatment. However, Rasmussen et al. (2020) concluded that low agreement between raters, particularly for process and relational skills, was a limitation of CAS-CBT in this setting. Contrary to this average good agreement between raters was found for adherence [ICC $(2,1)=0.63]$ and competence $[\operatorname{ICC}(2,1)=0.69]$ in the Haugland et al. (2020) study. These similarities and differences should have been discussed in view of differences in rater background, extensiveness of the programs, and statistical model chosen for the ICC. We believe that additional information from the previous study using the same instrument in a similar context (i.e., indicative prevention, group format, primary health setting) should have been included in the discussion of the findings in the Rasmussen et al. (2021) study.

\section{CONCLUSION}

The findings of the Rasmussen et al. study 2020 on reliability and applicability of CAS-CBT are scientifically important. However, their statement that this is the first study using CAS-CBT within an indicated prevention study is incorrect. Furthermore, a comparison with findings from a large previous RCT on indicated prevention for youth with anxiety, recruited and delivered within the same cultural context, would have broadened, and probably nuanced the discussion of the findings in Rasmussen et al. (2021) study.

\section{AUTHOR CONTRIBUTIONS}

BSMH and GJW wrote and edited the commentary.

\section{FUNDING}

The RCT-study was supported by The Research Council of Norway [Grant no. 229020]. Additional financial support was received from the Oslofjord fund [Grant no. 245807], Regional Research fund western Norway [Grant no. 235707], and the Norwegian Directorate of Health [Reference no. 11/751-38 and 14/4285-3].

Harstad, S., Bjaastad, J. F., Hjemdal, O., Compton, S., Waaktaar, T., and Aalberg, M. (2021). Competence and adherence scale for cognitive behavioural therapy (CAS-CBT) for anxiety disorders in youth: reliability and factor structure. Behav. Cogn. Psychother. 20:1-13. doi: 10.1017/S1352465821000217

Haugland, B. S. M., Haaland, Å. T., Baste, V., Bjaastad, J. F., Hoffart, A., Rapee, R. M., et al. (2020). Effectiveness of brief and standard school-based 
cognitive-behavioral interventions for adolescents with anxiety: a randomized non-inferiority study. J. Am. Acad. Child Adolescent Psychiatry 59, 552-564. doi: 10.1016/j.jaac.2019.12.003

Haugland, B. S. M., Raknes, S., Haaland, A. T., Wergeland, G. J., Bjaastad, J. F., Baste, V., et al. (2017). School-based cognitive behavioral interventions for anxious youth: study protocol for a randomized controlled trial. Trials 18:100. doi: 10.1186/s13063-017$1831-9$

Husabo, E., Haugland, B. S. M., McLeod, B. D., Baste, V., Haaland, Å. T., Bjaastad, J. F., et al. (2021). Treatment fidelity in brief versus standard-length school-based interventions for youth with anxiety. School Mental Health. doi: 10.1007/s12310-021-09458-2

Rasmussen, L.-M. P., Patras, J., Handegård, B. H., Neumer, S.-P., Martinsen, K. D., Adolfsen, F., et al. (2021). Evaluating delivery of a CBTbased group intervention for schoolchildren with emotional problems: examining the reliability and applicability of a video-based adherence and competence measure. Front. Psychol. 12:702565. doi: 10.3389/fpsyg.2021. 702565
Conflict of Interest: The authors declare that the research was conducted in the absence of any commercial or financial relationships that could be construed as a potential conflict of interest.

Publisher's Note: All claims expressed in this article are solely those of the authors and do not necessarily represent those of their affiliated organizations, or those of the publisher, the editors and the reviewers. Any product that may be evaluated in this article, or claim that may be made by its manufacturer, is not guaranteed or endorsed by the publisher.

Copyright (C) 2021 Haugland and Wergeland. This is an open-access article distributed under the terms of the Creative Commons Attribution License (CC BY). The use, distribution or reproduction in other forums is permitted, provided the original author(s) and the copyright owner(s) are credited and that the original publication in this journal is cited, in accordance with accepted academic practice. No use, distribution or reproduction is permitted which does not comply with these terms. 